\title{
Sampling Time Offset Estimation for IFDMA Uplink Systems
}

\author{
Alexander Arkhipov, Michael Schnell \\ German Aerospace Center (DLR), Inst. of Communications and Navigation, D-82234, Wessling, Germany. \\ Phone/e-mail: +49-8153-282873/firstname.lastname@dlr.de
}

\begin{abstract}
This article investigates the influence of sampling time offset on the performance of an interleaved frequencydivision multiple-access (IFDMA) uplink scheme for mobile radio transmission. It is shown that sampling time offset causes signal-to-noise ratio (SNR) degradation and leads to intersymbol interference (ISI). In addition, an efficient algorithm is proposed which is capable to estimate sampling time offset by applying oversampling at the receiver. The proposed algorithm requires only three-times oversampling, does not use any pilot symbols, and is independent from the transmission channel and modulation alphabet.
\end{abstract}

\section{INTRODUCTION}

Recently, IFDMA attained significant importance and is considered as an air interface candidate for $4 \mathrm{G}$ mobile communications [3]. IFDMA is equivalent to an orthogonal frequencydivision multiple-access code-division multiplexing (OFDMACDM) [2] scheme with block interleaved frequency allocation and Fourier spreading [11] [7]. Compared to conventional OFDMA [1], IFDMA seems more attractive due to its lower complexity, since IFDMA requires no discrete Fourier transform (DFT) at the transmitter. Moreover, IFDMA shows a significantly lower peak-to-average power ratio (PAPR) than conventional OFDMA.

If a transmit filter is applied, the correct sampling at the receiver becomes important. Several studies have been accomplished to estimate the time offset for OFDMA uplink systems [6] [9]. In these works, rectangular pulse shaping is assumed, and the problem of correct sampling is not considered. In contrast to OFDMA, rectangular pulse shaping is not an appropriate choice, since this pulse-shaping at the IFDMA transmitter results in an inefficient power density spectrum. Therefore, a transmit filter should be applied in the case of IFDMA.

Standard sampling time offset estimation algorithms for singlecarrier systems [10] cannot be applied to an IFDMA receiver. First, in an IFDMA receiver, equalization is preferably performed in the frequency domain. Second, the influence of a time-dispersive mobile radio channel must be taken into account. Third, received uplink signals from different users are distorted by different transmission channels.

In this article, we investigate the performance of an IFDMA uplink system for mobile radio transmission in the presence of sampling time offset misalignment and propose an algorithm for sampling time offset estimation. We assume that sampling time offset is equal or less than the chip duration. The base station receives data from different users simultaneously and, ideally, all users are perfectly aligned in time. However, in our investigation, we consider that all users can have random time delays, which have to be less than the chip duration.

The obtained estimates of the sampling time offsets of each user can be used for the correcting of sampling time instances at the base station receiver, or to transmit time correction values back to the mobile terminals in order to fine-tune users in time.

We use the following notation in this article: variables denoting signals in time domain are written in small letters, variables denoting signals in frequency domain are written in capital letters. Bold variables designate matrices or vectors.

The remainder of this article is organized as follows. In section II IFDMA scheme is briefly renewed. The influence of sampling time offset on the system performance is studied in section III. Section IV describes the proposed sampling time offset estimation algorithm and simulation results are discussed in section V. Finally, section VI concludes this article.

\section{INTERLEAVED FREQUENCY-DIVISION MULTIPLE ACCESS SCHEME}

In the following, IFDMA is described as a special case of OFDMA-CDM. For additional descriptions of the IFDMA scheme refer e.g to [5] [4]. The standard OFDMA-CDM [2] uplink transmission system is able to support up to $K$ simultaneously active users. Assume that $N_{u}$ users $\left(N_{u} \leq\right.$ $K)$ transmit simultaneously. Each OFDMA-CDM user, $i=$ $1, \ldots, N_{u}$, performs a blocked transmission of $Q$ complexvalued symbols $d_{q}^{(i)}, q=1, \ldots, Q$, which are assumed to be equally likely and are taken from the underlying modulation alphabet. The average energy of the transmitted data symbol $d_{q}^{(i)}$ is unity and the symbol duration is $T_{s}$. The $Q$ symbols are arranged to build the symbol vector $\mathbf{d}^{(i)}$ of user $i$

$$
\mathbf{d}^{(i)}=\left(d_{1}^{(i)}, d_{2}^{(i)}, \ldots, d_{Q}^{(i)}\right)^{T}
$$

The symbol vector $\mathbf{d}^{(i)}$ is first serial-to-parallel converted. Then, a code-division multiplexing (CDM) component is introduced by spreading each symbol with a different spreading code $\mathbf{c}_{q}, q=1, \ldots, Q$, of length $L \geq Q$ and adding together the resulting $Q$ spread vectors. After summation, we obtain vector $\mathbf{s}^{(i)}$ which is referred as a vector of the spread symbols. It can be represented by its components $s_{n}^{(i)}, n=0, \ldots, L-1$ 


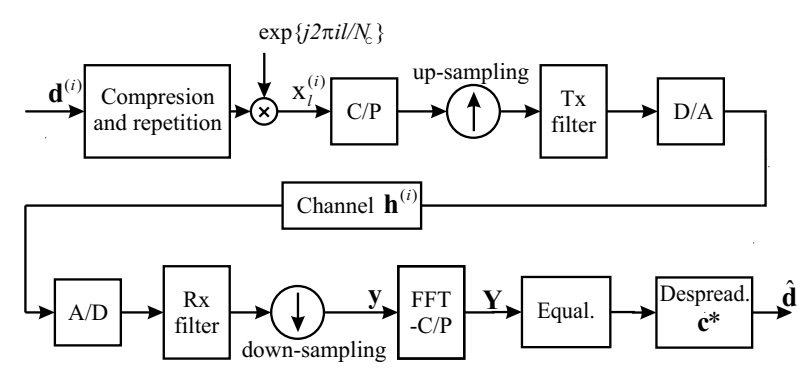

Fig. 1. Simulation model for IFDMA uplink

as

$$
s_{n}^{(i)}=\sum_{q=1}^{Q} c_{n, q} \cdot d_{q}^{(i)}, n=0, \ldots, L-1,
$$

where $c_{n, q}$ is the $n$th component of the spreading code $\mathbf{c}_{q}$. Finally, an inverse DFT (IDFT) with user-specific frequency mapping is performed for the vector $\mathbf{s}^{(i)}$. Block interleaving achieves optimal frequency diversity, since it distributes the $L$ spread symbols $s_{n}^{(i)}$ of the $Q$ data symbols of user $i$ equally over the whole transmission bandwidth. Note, OFDMA-CDM exclusively assigns to each of the $K$ users a set of $L$ frequencies out of all possible $N_{c}=K L$ subcarriers. If all subcarriers assigned to user $i$ are uniformly spaced over the whole available transmission bandwidth at a distance $N_{c} / K$, the time domain transmit signal $x_{l}^{(i)}, l=0, \ldots N_{c}-1$, obtained after the IDFT operation in the transmitter, can be represented by

$$
x_{l}^{(i)}=e^{j \frac{2 \pi}{N_{c}} i l} \frac{1}{\sqrt{N_{c}}} \sum_{n=0}^{L-1} s_{n}^{(i)} e^{j \frac{2 \pi}{L} n l} .
$$

As in standard OFDMA, a guard interval larger than the maximum channel delay is added as a cyclic prefix (CP) in OFDMA-CDM in order to avoid interference from preceding OFDMA-CDM transmission symbols.

IFDMA as a special case of OFDMA-CDM can be realized if a fully loaded system, i.e. $L=Q$, is considered and Fourier codes with components $c_{n, q}=\left\{e^{-j \frac{2 \pi}{Q} n q}\right\}, n=0, \ldots, L-$ $1, q=1, \ldots, Q$, are used for spreading. In this case, the Fourier codes for spreading and the rotation factors of the Fourier transform cancel out and the transmit signal given in (3) reduces to

$x_{l}^{(i)}=\underbrace{e^{\frac{j 2 \pi}{N_{c}} i l}}_{\text {user specific phase factor }} \cdot \frac{1}{\sqrt{K}} d_{l \bmod L}^{(i)}, l=0, \ldots, N_{c}-1$.

Comparing (4) and the IFDMA signal generation as described in [5] [4] it follows that IFDMA is equivalent to OFDMACDM with block interleaved frequency allocation and can be completely generated in the time domain without using the Fourier transform. The time domain generation of IFDMA uses compression and repetition as described in detail in [5]. Note, the multiplication with the user specific phase factor in (4) ensures the user discrimination by assigning to each user $i$ a set of subcarriers orthogonal to all other users' subcarrier sets. Each sample $x_{l}^{(i)}$ represents the transmission chip $l, l=0, \ldots, N_{c}-1$. The duration of the resulting chips is $T_{c}=T_{s} / K$.

The simulation model for IFDMA is shown in Fig. 1. After addition of CP, the obtained signal is up-sampled. Up-sampling is implemented by adding zero samples between successive samples $x_{l}^{(i)}$. After up-sampling, pulse-shaping is performed by a transmit filter. After digital-to-analog (D/A) conversion, we assume transmission over a time-dispersive mobile radio channel. At the receiver, we consider oversampling per transmitted chip $x_{l}^{(i)}$. After sampling, the signal is filtered by a receive filter, which is matched to the transmit pulse-shaping filter. In Fig. 2. an example of the IFDMA signal after the receive filter is represented. The solid lines represent the pulseshapes corresponding to the different chips and the dotted line is the sum signal. In the down-sampling operation at least one sample per transmitted chip is chosen for performing equalization, despreading and estimation of the transmitted data.

For the sake of simplicity, the convolution of the receive and transmit filters is represented by the function $g(t)$, which is chosen as a Nyquist pulse

$$
g(t)=\operatorname{sinc}\left(\frac{t}{T_{c}}\right) \frac{\cos \left(\pi \alpha t / T_{c}\right)}{1-4 \alpha^{2} t^{2} / T_{c}^{2}}
$$

where $\alpha$ denotes the roll-off factor.

In this section only one receive sample $y_{l}, l=0, \ldots, N_{c}-1$ per transmitted chip $x_{l}^{(i)}$ is considered. We assume that the channel has an impulse response described by the vector $\mathbf{h}^{(i)}$ of dimension $M$ with components $h_{m}^{(i)}, m=1, \ldots, M$. Without loss of generality, we assume that $N_{c}>M$, in the following.

Assume that $y_{l}$ represents the $l$ th element of the received vector $\mathbf{y}$, which can be written as

$$
\mathbf{y}=\sum_{i=1}^{N_{u}} \hat{\mathbf{h}}^{(i)} \mathbf{z}^{(i)}\left(\tau^{(i)}\right)+\mathbf{n}
$$

where the vector $\mathbf{n}$ denotes the samples of the additive white Gaussian noise (AWGN). The channel matrix $\hat{\mathbf{h}}^{(i)}$

$$
\hat{\mathbf{h}}^{(i)}=\left(\begin{array}{cccccc}
h_{1}^{(i)} & \ldots & h_{M}^{(i)} & 0 & \ldots & 0 \\
0 & h_{1}^{(i)} & \ldots & h_{M}^{(i)} & \ldots & 0 \\
\vdots & \vdots & \ddots & \vdots & \vdots & \vdots \\
0 & \ldots & h_{1}^{(i)} & \ldots & h_{M}^{(i)} & 0 \\
0 & 0 & \ldots & h_{1}^{(i)} & \ldots & h_{M}^{(i)}
\end{array}\right)
$$

is the $N_{c} \times N_{c}$ right circular matrix whose rows are cyclically shifted versions of the vector $\tilde{\mathbf{h}}^{(i)}$. The vector $\tilde{\mathbf{h}}^{(i)}$ is obtained by appending $N_{c}-M$ zeros to $\mathbf{h}^{(i)}$.

The elements $z_{l}^{(i)}\left(\tau^{(i)}\right)$ of vector $\mathbf{z}^{(i)}\left(\tau^{(i)}\right)$ in (6) can be 


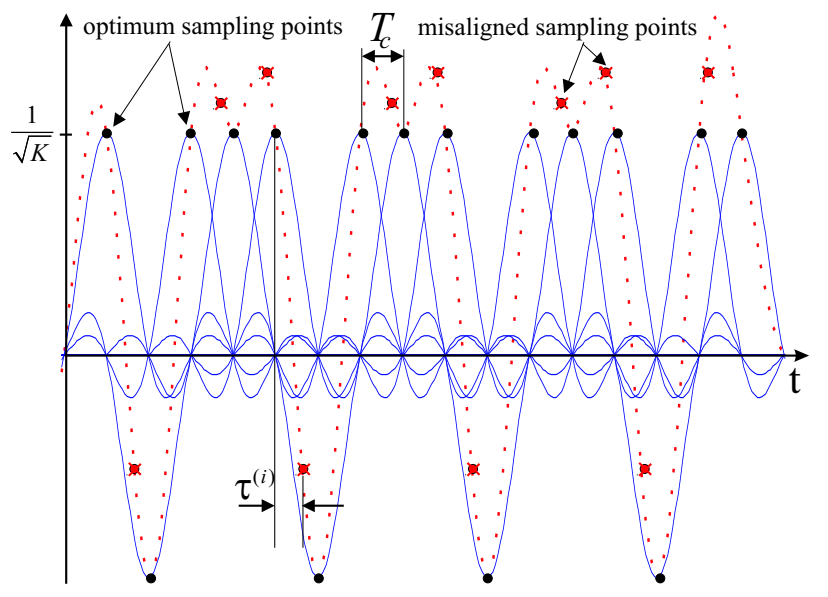

Fig. 2. Example of the IFDMA signal after the receive filter. Sampling time offset $\tau^{(i)}$ causes SNR degradation and leads to ISI; $\mathbf{d}^{(i)}=[1,-1,1,1]^{T}$; $L=Q=4 ; \alpha=0.25$; only user $i=0$ is active; AWGN channel with a negligible noise power.

represented as

$$
\begin{gathered}
z_{l}^{(i)}\left(\tau^{(i)}\right)=e^{j \frac{2 \pi}{N_{c}} i\left(l+\frac{\tau^{(i)}}{T_{c}}\right)} \frac{1}{\sqrt{K}} \cdot \\
{[\underbrace{d_{l \text { mod } L}^{(i)} g\left(\tau^{(i)}\right)}_{\text {useful signal }}+\underbrace{\sum_{\substack{p=0 \\
p \neq l}}^{N_{c}-1} d_{p \bmod L}^{(i)} g\left(\tau^{(i)}+(p-l) T_{c}\right)}_{\text {ISI }}] .}
\end{gathered}
$$

The normalized time offset $\tau^{(i)}$ can take on values in the range $-T_{c} / 2 \leq \tau^{(i)} \leq T_{c} / 2$. The orthogonality between users is not violated since $\tau^{(i)}$ does not introduce any frequency offset. However, ISI appears if a sampling time offset $\tau^{(i)}$ is present as shown in Fig. 2, The optimum sampling points correspond to a situation where $\tau^{(i)}=0$. In this case, the received signal of user $i$ is ISI-free.

Defining $\mathbf{W}$ as a $N_{c} \times N_{c}$ DFT matrix, the frequency domain representation $\mathbf{Y}$ of the received signal $\mathbf{y}$ can be written as

$$
\mathbf{Y}=\sum_{i=1}^{N_{u}} \mathbf{H}^{(i)} \mathbf{Z}^{(i)}\left(\tau^{(i)}\right)+\mathbf{N}
$$

where $\mathbf{H}^{(i)}=\mathbf{W} \hat{\mathbf{h}}^{(i)}$ is a $N_{c} \times N_{c}$ diagonal matrix, which is the DFT transformation of the channel impulse response $\hat{\mathbf{h}}^{(i)}$. The received signal of user $i$ in the frequency domain is defined as $\mathbf{Z}^{(i)}\left(\tau^{(i)}\right)=\mathbf{W} \mathbf{z}^{(i)}\left(\tau^{(i)}\right)$ and $\mathbf{N}=\mathbf{W} \mathbf{n}$.

The vector $\mathbf{Y}$ can be used for the estimation of the transmitted data. User $i$ transmits its $L$ spread symbols $s_{n}^{(i)}$ on subcarriers $\kappa=n K+i, n=0, \ldots, L-1$. Other users transmit their data on separated orthogonal subcarriers and their influence is not considered in the following. Thus, we omit subscript $i$ for simplicity and focus on the data of user $i$. After frequency domain equalization, the $L$ received values $Y_{\kappa}$ can be despread with the complex-conjugate Fourier codes (IDFT operation of length $L$ ) to create an estimation $\hat{\mathbf{d}}$ of the symbol vector $\mathbf{d}$. The considered receiver is equivalent to the conventional OFDMACDM receiver [2], only Fourier codes are used instead of Walsh-Hadamard codes.

\section{ISI AND SNR DEGRADATION DUE TO SAMPLING TIME OFFSET}

We consider vector $\mathbf{z}(\tau)$ in our analysis of ISI. ISI appears if a sampling time offset $\tau$ is present as given in (8). If $L$ is large enough, the variance of the ISI for chip $x_{l}$ can be approximated as

$$
\begin{aligned}
\sigma_{\text {ISI }}^{2}(\tau) & =E\left\{\left|\frac{1}{\sqrt{K}} \sum_{\substack{p=0 \\
p \neq l}}^{N_{c}-1} d_{p \bmod L} g\left(\tau+(p-l) T_{c}\right)\right|^{2}\right\}(10) \\
& =\frac{1}{K} \sum_{\substack{p=0 \\
p \neq l}}^{N_{c}-1} g^{2}\left(\tau+(p-l) T_{c}\right)
\end{aligned}
$$

where the operator $E\{$.$\} defines expectation. The energy of$ the useful signal in (8) decreases with $\tau$ according to

$$
E\left\{\left|d_{l \bmod L} g(\tau)\right|^{2}\right\} / K=g^{2}(\tau) / K .
$$

Additional phase rotation in (8) can be mitigated by a channel estimation algorithm and does not have any negative impact on the system performance.

\section{TIME OFFSET ESTIMATION}

Our goal is to estimate the time offset $\tau$ without any additional pilot symbols. In the following, we propose an algorithm which allows to estimate $\tau$ using tree times oversampling per transmitted chip. Thus, we consider the case where sampling is performed with a period $\frac{T_{c}}{3}$ and in addition to vector $\mathbf{y}$ we introduce two vectors $\mathbf{y}^{\prime}$ and $\mathbf{y}^{\prime \prime}$. If we omit the influence of other users, $\mathbf{y}^{\prime}$ and $\mathbf{y}^{\prime \prime}$ can be defined as

$$
\mathbf{y}^{\prime}=\hat{\mathbf{h}} \mathbf{z}\left(\tau-\frac{T_{c}}{3}\right)+\mathbf{n}^{\prime}
$$

and

$$
\mathbf{y}^{\prime \prime}=\hat{\mathbf{h}} \mathbf{z}\left(\tau+\frac{T_{c}}{3}\right)+\mathbf{n}^{\prime \prime},
$$

respectively. We introduce also two additional frequency domain vectors $\mathbf{Y}^{\prime \prime}=\mathbf{W} \mathbf{y}^{\prime \prime}$ and $\mathbf{Y}^{\prime}=\mathbf{W} \mathbf{y}^{\prime}$. Note, only the elements $Y_{\kappa}^{\prime}$ and $Y_{\kappa}^{\prime \prime}, \kappa=n K+i, n=0, \ldots, L-1$ of vectors $\mathbf{Y}^{\prime}$ and $\mathbf{Y}^{\prime \prime}$ represent data of user $i$.

Thus, we have three frequency domain vectors $\mathbf{Y}, \mathbf{Y}^{\prime}$ and $\mathbf{Y}^{\prime \prime}$. The expectations of the elements of these vectors $Y_{\kappa}, Y_{\kappa}^{\prime}$ and $Y_{\kappa}^{\prime \prime}$ are given as

$$
\begin{gathered}
E\left\{\left|Y_{\kappa}\right|^{2}\right\}=E\left\{\left|H_{\kappa, \kappa}\right|^{2}\right\} E\left\{\left|Z_{\kappa}(\tau)\right|^{2}\right\}+\sigma_{n}^{2} \\
E\left\{\left|Y_{\kappa}^{\prime}\right|^{2}\right\}=E\left\{\left|H_{\kappa, \kappa}\right|^{2}\right\} E\left\{\left|Z_{\kappa}\left(\tau-\frac{T_{c}}{3}\right)\right|^{2}\right\}+\sigma_{n}^{2}
\end{gathered}
$$


and

$$
E\left\{\left|Y_{\kappa}^{\prime \prime}\right|^{2}\right\}=E\left\{\left|H_{\kappa, \kappa}\right|^{2}\right\} E\left\{\left|Z_{\kappa}\left(\tau+\frac{T_{c}}{3}\right)\right|^{2}\right\}+\sigma_{n}^{2},
$$

where $\sigma_{n}^{2}$ is the variance of the filtered noise, $Z_{\kappa}(\tau), Z_{\kappa}(\tau-$ $\left.\frac{T_{c}}{3}\right)$ and $Z_{\kappa}\left(\tau+\frac{T_{c}}{3}\right)$ are the components of vectors $\mathbf{Z}(\tau)$, $\mathbf{Z}\left(\tau-\frac{T_{c}}{3}\right)$ and $\mathbf{Z}\left(\tau+\frac{T_{c}}{3}\right)$ taken at $\kappa=n K+i, n=0, \ldots, L-1$. The fading coefficients $H_{\kappa, \kappa}, \kappa=n K+i, n=0, \ldots, L-1$ are the diagonal elements of $\mathbf{H}$. It can be noted that $H_{\kappa, \kappa}$ are the same in (14), (15) and (16). This assumption can be considered as realistic, since system design rules assume that fading coefficients remain constant over a duration of one IFDMA symbol, i.e, during a time period equal to $N_{c} T_{c}$.

The expectations $E\left\{\left|Y_{\kappa}\right|^{2}\right\}, E\left\{\left|Y_{\kappa}^{\prime}\right|^{2}\right\}$ and $E\left\{\left|Y_{\kappa}^{\prime \prime}\right|^{2}\right\}$ can be approximated as averages of the energies of the received data $Y_{\kappa}, Y_{\kappa}^{\prime}$ and $Y_{\kappa}^{\prime \prime}$. For example, such an averaging can be performed over the whole transmission frame.

The DFT operation does not change the energy of the signal, i.e. $E\left\{\left|Z_{\kappa}(\tau)\right|^{2}\right\}=K \cdot E\left\{\left|z_{l}(\tau)\right|^{2}\right\}$. Therefore, the value $E\left\{\left|Z_{\kappa}(\tau)\right|^{2}\right\}$ can be approximated as

$$
E\left\{\left|Z_{\kappa}(\tau)\right|^{2}\right\}=E_{s}(\tau)=\sum_{p=-N_{c} / 2}^{N_{c} / 2} g^{2}\left(\tau+p T_{c}\right) .
$$

Taking into account (14), (15), (16) and (17) and introducing new variables $A=E\left\{\left|Y_{\kappa}\right|^{2}\right\}-E\left\{\left|Y_{\kappa}^{\prime \prime}\right|^{2}\right\}, B=E\left\{\left|Y_{\kappa}^{\prime}\right|^{2}\right\}$ $E\left\{\left|Y_{\kappa}\right|^{2}\right\}$ and $C=E\left\{\left|Y_{\kappa}^{\prime \prime}\right|^{2}\right\}-E\left\{\left|Y_{\kappa}^{\prime}\right|^{2}\right\}$, the estimate $\hat{\tau}$ of the normalized time offset $\tau$ can be found as

$$
\begin{aligned}
& \hat{\tau}= \\
& \quad \arg \min _{\tilde{\tau}}\left|A E_{s}\left(\tilde{\tau}-\frac{T_{c}}{3}\right)+B E_{s}\left(\tilde{\tau}+\frac{T_{c}}{3}\right)+C E_{s}(\tilde{\tau})\right| .
\end{aligned}
$$

The following remarks are of interest:

- The estimation in (18) does not require the knowledge of the transmission channel and the modulation alphabet.

- The estimation in (18) can be performed only if $\frac{T_{c}}{2} \leq$ $\tau \leq \frac{T_{c}}{2}$, since function $E_{s}(\tau)$ is periodic with a period of $T_{c}$.

- The proposed estimation requires only one dimensional search over possible values of $\tau$. Values $E_{s}(\tau)$ can be precalculated at the receiver according to formula (17).

One of the vectors $\mathbf{Y}, \mathbf{Y}^{\prime}$ and $\mathbf{Y}^{\prime \prime}$ can be used for despreading and data demodulation.

\section{Simulation Results}

The transmission system considered for simulations has a bandwidth of $20 \mathrm{MHz}$ and the carrier frequency of $5 \mathrm{GHz}$. The number of subcarriers is $N_{c}=1024$. The spreading length is $L=64$ and the total number of users is $K=16$. A minimum mean square error (MMSE) detector is used as described in [2]. For the channel coding, a convolutional code with rate $1 / 2$ and memory 6 is used in our simulations. The transmission frame consists of 24 IFDMA symbols. QPSK is used for modulation. Influence of the receive and the transmit filter is modelled as the Nyquist pulse $g(t)$, which assumed to be

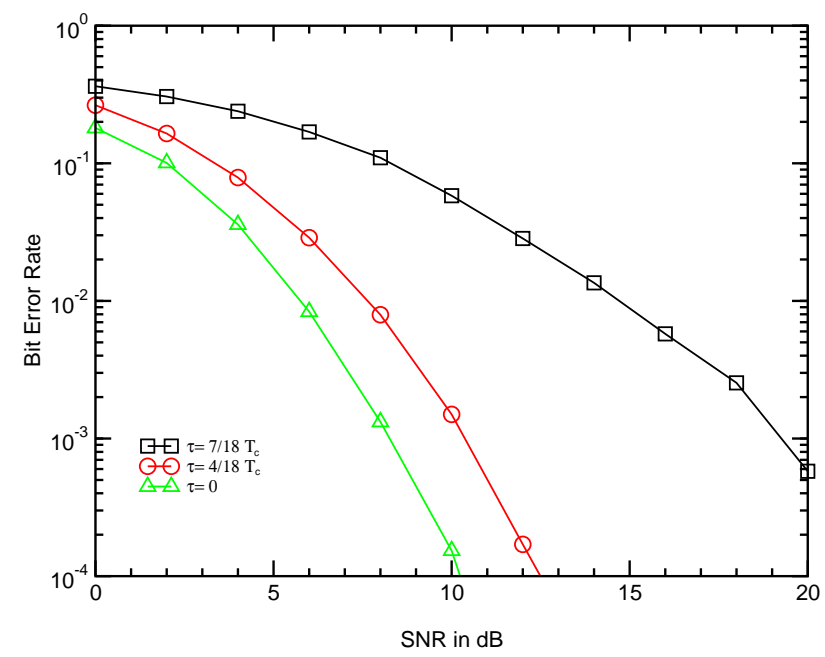

Fig. 3. BER versus SNR for the different values of sampling time offset $\tau ; \alpha=0.25$

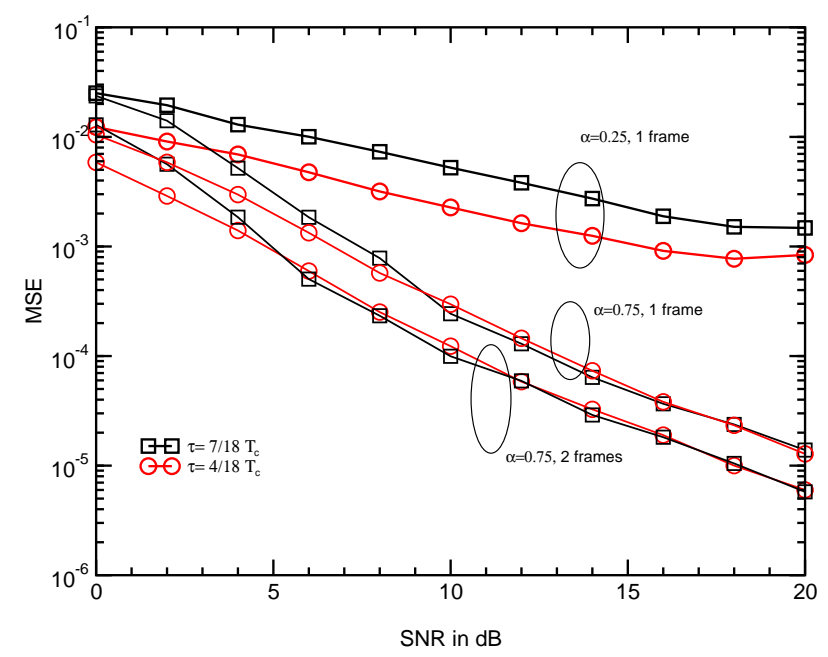

Fig. 4. MSE of the sampling time offset estimate versus SNR

non-zero only within $-3 T_{c}<t<3 T_{c}$. Therefore, if sampling time offset is present, each transmitted chip $x_{l}$ experiences ISI from three preceding and three following chips. The roll-off factor $\alpha$ varies from 0.25 to 0.75 .

A multipath channel model is considered, which consists of a tapped delay line with $M=11$ statistically independent Rayleigh fading taps. The average power for each channel component $h_{m}, m=1, \ldots, M$, is modelled as

$$
E\left\{\left|h_{m}\right|^{2}\right\}=-m+1(\mathrm{~dB}) .
$$

Additionally, the average channel attenuation is normalized to unity in our simulations.

We evaluate the system performance in terms of bit-error rate (BER) and mean square error (MSE) of the sampling time offset estimate. In Fig. 3 the system performance versus SNR is present for sampling time offsets $\tau=\frac{4}{18} T_{c}$ and $\tau=\frac{7}{18} T_{c}$. As a reference, the ideal case with $\tau=0$ is presented. It 
is shown that the performance of the system degrades with increasing $\tau$, due to the increase of ISI and SNR degradation. However, SNR degradation does not play a significant role in the performance loss. According to (11), SNR degradation is $-0.74 \mathrm{~dB}$ for $\tau=\frac{4}{18} T_{c}$ and $-2.36 \mathrm{~dB}$ for $\tau=\frac{7}{18} T_{c}$. Therefore, ISI is responsible for the residual performance degradation.

The MSE of the sampling time offset estimation in (18) is presented in Fig. 4 for $\tau=\frac{4}{18} T_{c}$ and $\tau=\frac{7}{18} T_{c}$ as well as for different values of $\alpha$ equal to 0.25 and 0.75 . For $\alpha=0.25$, the performance of the proposed algorithm degrades if $\tau$ increases. In contrast, the performance of the algorithm is independent from $\tau$ if $\alpha=0.75$. Close inspection of the function $E_{s}(\tau)$ explains this fact. If $\alpha=0.25$, the function $E_{s}(\tau)$ is relatively flat. Hence, the function in (18) does not have a distinct minimum, and the obtained estimation is easily affected by noise. The situation changes completely, if we increase $\alpha$. The difference between the maximum and minimum value of $E_{s}(\tau)$ is nearly two times larger for $\alpha=0.75$ than for $\alpha=0.25$. If $\alpha=0.75$, the function $E_{s}(\tau)$ increases steeply and this steepness remains nearly constant with increasing $\tau$. Therefore, MSE is independent of $\tau$ for $\alpha=0.75$. How it is seen from Fig. 4, the required SNR to achieve a MSE of $10^{-3}$ is $14 \mathrm{~dB}$ less for $\alpha=0.75$ than for $\alpha=0.25$.

The precision of the estimate can be improved if averaging in (14), (15) and (16) is performed over the frame with a large size or over many frames. In Fig. 4 this averaging is performed over two frames for the case of $\alpha=0.75$. It can be seen that the performance is improved by $3 \mathrm{~dB}$. However, such an improved averaging leads to a delay in the sampling time offset estimation and is not desirable.

\section{CONCLUSIONS}

The performance of the IFDMA uplink system is investigated in the presence of sampling time offset. It has been shown that sampling time offset results in ISI and SNR degradation. Moreover, we have proposed an algorithm for sampling time offset estimation using three times oversampling at the receiver. The proposed algorithm is independent from the transmission channel and modulation alphabet.

\section{ACKNOWLEDGMENTS}

The work presented in this article is carried out in the framework of the 4MORE (4G MC-CDMA multiple antenna system On chip for Radio Enhancements) project [8], which is supported by the European Commission within FP6 under the contract number IST-2002-507039. The authors would like to acknowledge this support and this opportunity to conduct the research work.

[1] R.V. Nee and R. Prasad, OFDMA for Wireless Multimedia Communications, Artech House, 2002.

[2] S. Kaiser, "OFDM code division multiplexing in fading channels", IEEE Transactions on Communications, vol. 50, no. 8, pp. 1266-1273, Aug. 2002.

[3] H. Atarashi and M. Sawahashi, "Broadband wireless access based on VSF-OFCDM and VSCRF-CDMA and its experiments", MultiCarrier Spread Spectrum \& Related Topics (MC-SS), Oberpfaffenhofen, Germany, Sept. 2003.
[4] U. Sorger, I.De Broeck, and M. Schnell, "Interleaved FDMA - a new spread-spectrum multiple-access scheme", IEEE International Conference on Communications (ICC'98), pp. 1013-1017, June.1998.

[5] M. Schnell, I. De Broeck, and U. Sorger, "A promising new wideband multiple-access scheme for future mobile communications systems", European Transactions on Telecommunications (ETT), vol. 10, No. 4, pp. 417-427, Jul./Aug. 1999.

[6] M. Morelli, "Timing and frequency synchroniozation for the uplink of an OFDMA system", IEEE Transactions on Communications, vol. 52, no. 2, pp. 286-306, Feb. 2004.

[7] K. Bruninghaus and H. Rohling, "Multi-carrier spread-spectrum and its relation to single-carrier transmission", IEEE Vehicular Technology Conference (VTC-Spring 98), Ottawa, Canada, 1998.

[8] IST 4MORE project, web site http://www.ist-4more.org

[9] M. Park, K.Ko, H.Yoo, and D.Hong, "Performance analysis of OFDMA uplink systems with symbol timing misallignment", IEEE Communications Letters, vol. 7, pp. 376-379, Aug. 2003.

[10] J. Proakis, Digital Communications, McGraw Hill Higher Education, Dec. 2000.

[11] A. Arkhipov and M.Schnell, "Interleaved frequency-division multipleaccess system with frequency domain equalization", 9th. International OFDM Workshop (InOWo'06), Dresden, Germany, Sep. 2004. 